DrAFt VERSION June 25, 2019

Typeset using $\mathrm{LAT}_{\mathrm{E}} \mathrm{X}$ preprint2 style in AASTeX61

\title{
QUARK NOVAE: AN ALTERNATIVE CHANNEL FOR THE FORMATION OF ISOLATED MILLISECOND PULSARS
}

\author{
Nurimangul Nurmamat, ${ }^{1}$ Chunhua Zhu, ${ }^{1}$ Guoliang Lü, ${ }^{1}$ Zhaojun Wang, ${ }^{1}$ Lin Li, ${ }^{1}$ And \\ HELEI LIU ${ }^{1}$ \\ ${ }^{1}$ School of Physical Science and Technology, Xinjiang University, Urumqi, 830046, China
}

\begin{abstract}
Isolated millisecond pulsars (IMSPs) are a topic of academic contention. There are various models to explain their formation. We explore the formation of IMSP via quark novae (QN). During this formation process, low-mass X-ray binaries (LMXBs) are disrupted when the mass of the neutron star (NS) reaches $1.8 \mathrm{M}_{\odot}$. Using population synthesis, this work estimates that the Galactic birthrate of QN-produced IMSPs lies between $\sim 9.5 \times 10^{-6}$ and $\sim 1.7 \times 10^{-4} \mathrm{yr}^{-1}$. The uncertainties shown in our experiment model is due to the QN's kick velocity. Furthermore, our findings not only show that QN-produced IMSPs are statistically more significant than those produced by mergers, but also that millisecond pulsar binaries with a high eccentricity may originate from LMXBs that have been involved in, yet not disrupted by, a QN.
\end{abstract}

Keywords: pulsars: general- stars: neutron- binaries: close

Corresponding author: Chunhua Zhu

chunhuazhu@sina.cn

nurimangul_uy@sina.com 


\section{INTRODUCTION}

Millisecond Pulsars (MSPs) are a particular type of pulsar, well-known for their old age. They differ from normal pulsars, not only due to their short spin period $(\leq 30 \mathrm{~ms})$ but also because of their low magnetic field $\left(\sim 10^{8} \mathrm{G}\right)$ (e.g., Lorimer et al. 1996; Tauris 2016). In most cases, normal pulsars have a long slow-down rates $\left(\dot{P} \sim 10^{-15}\right)$, whereas the slow-down rate of MSPs is usually $\dot{\mathrm{P}} \sim 10^{-19}$. MSPs stable rotations and sharp radio pulses make them exceptionally stable clocks (Rawley et al. 1987; Backer 1987), being used by the International Pulsar Timing Array Project to detect gravitational waves(Manchester \& IPTA 2013).

MSPs are usually formed in low- and intermediatemass X-ray binaries through a process of recycling (Alpar et al. 1982), whereby Roche lobe overflow from its companion star causes a neutron star (NS) to not only accrete mass $\left(\geq 0.1 \mathrm{M}_{\odot}\right)$ and angular momentum over a lengthy period of time $\left(\sim 10^{8} \mathrm{yr}\right)$, but also increase in rotational speed up to the millisecond (Alpar et al. 1982; Bhattacharya \& Srinivasan 1991; van den Heuvel 2008). This may lead one to surmise that the majority of MPSs have evolved from and exist solely in binary systems. However, observational data from the Pulsar Catalogue at ATNF (Australia Telescope National Facility) shows a total of 360 MSPs, approximately 120 of which exist in isolation (Manchester et al. 2005). These have been called Isolated Millisecond Pulsars (IMSPs) (van den Heuvel \& van Paradijs 1988).

The origin of IMSPs is a topic of academic contention. Investigations undertaken by van den Heuvel \& Bonsema (1984) have suggested that a close binary system, not only consisting of a NS and a massive white dwarf (WD), but also with a $\mathrm{P}_{\text {orb }}<16$ hours, may inescapably merge into an IMSP, as a consequence of gravitational radiation loss $\left(\mathrm{M}>0.7 \mathrm{M}_{\odot}\right)$. For such NSs and massive
WDs $\left(\right.$ ¿0.9M $\left._{\odot}\right)$, Davies et al. (2002) have proposed a formation rate of $5 \times 10^{-4} \sim 5 \times 10^{-5}$ $\mathrm{yr}^{-1}$. Ferrario \& Wickramasinghe (2007) and Grégoire \& Knödlseder (2013), however, have suggested that the formation rate of IMSPs ranges from $3 \times 10^{-6} \sim 5 \times 10^{-6} \mathrm{yr}^{-1}$. This figure is significantly lower than the Galactic birth rate of IMSP formed through the merging of massive WDs and NSs, hence rendering it somewhat unreliable. Whats more, in recent literature, Sun et al. (2018) used population synthesis to investigate the possible origin of IMSPs as product of NS and WD coalescence, putting forward the estimate that IMSPs Galactic birthrate may lie between $5.8 \times 10^{-5} \mathrm{yr}^{-1}$ and $2.0 \times 10^{-4} \mathrm{yr}^{-1}$.

In Camilo et al. (1993), it is suggested that IMSPs may be formed after recycled high-mass $\mathrm{X}$-ray binary systems are disrupted by the evolution of high-mass companion stars into supernovae. However, according to data from Belczynski et al. (2010), only $10 \%$ of disrupted recycled pulsars are IMSPs. For this reason, one can infer that disrupted recycled pulsars play but a small part in the formation of IMSPs.

Portegies Zwart et al. (2011) put forward a triple-star theory to explain the formation of MSPs that are both in binaries with normal stars and have wide orbits with a high eccentricity, such as J1903+0327. Their results indicated that MSPs can be ejected from triple-star systems, due to their dynamic instability, thereby bringing about the formation of IMSPs. Despite this, the amount of IMSPs produced this way seems somewhat insignificant when compared to their overall number.

According to Kluzniak et al. (1988), loss of mass in a binary system comprised of an MSP and a low-mass companion star may be due to low-frequency electromagnetic radiation, energetic particles, or $\gamma$-rays produced by the pulsars fast-spinning magnetic dipole. Building on this theory, Ruderman et al. (1989) then sug- 
gested that if such irradiation can effectively evaporate the companion star, then it is possible that it can be entirely ablated, hence forming an IMSP. However, research by Stappers et al. (1998) Chen et al. (2013) and Lü et al. (2017) have estimated that this would take a significantly long time to occur, rendering it extremely difficult to produce IMSPs within the confines of hubble time.

Here, this paper shall propose an alternative theory for the formation of IMSPs. At first, Witten (1984) acknowledged the existence of compact objects made of strange quark matter, called strange stars (SSs). Cheng \& Dai (1996) then noted that these SSs were formed in low-mass X-ray binaries (LMXBs) during an accretion-triggered transition phase, which has been called a quark nova (QN)(Ouyed et al. 2002). Similar to core collapse supernovae, SSs also gain post-QN kick velocity(Ouyed et al. 2002; Keränen et al. 2005; Ouyed et al. 2011, 2013, 2015). Thus, LMXBs are disrupted and IMSPs are formed.

In the following sections, this paper shall investigate the possibility of QN-induced IMSP formation. In $\S 2$ below, we present our hypothesis and provide some details of our modelling algorithm. This is then followed by a discussion of the results and effects of different parameters in $\S 3$. Finally, $\S 4$, presents our main conclusions.

\section{MODELS}

In this work, we employ the rapid binary star evolution code (BSE), which was first explored by Hurley et al. (2002) and then further expanded upon by Kiel \& Hurley (2006). Up to the present, our research team have used the BSE code to investigate several types of binary systems, including symbiotic stars (Lü et al. 2006, 2009, 2011), X-ray binaries (Lü et al. 2012, 2017; Zhu et al. 2017), accreting MSPs (Zhu et al. 2015), classical nova (Sun et al. 2016; Rukeya et al. 2017), runaway stars pro- duced by supernovae (Yisikandeer et al. 2016), and Thorne-Zytkow objects (Hutilukejiang et al. 2018). In particular, Zhu et al. (2013) used the BSE code to examine QN-induced SS formation, but did not consider the role of kick velocity. This paper adopts the model previously presented by Zhu et al. (2013), not only taking into consideration three different means of NS production (core-collapse supernovae, evolutioninduced collapses and accretion-induced collapses), but also the increasing mass of the accreting NS. Following Zhu et al. (2013), this work also assumes that a $\mathrm{QN}$ occurs when the mass of an NS reaches $\sim 1.8 M_{\odot}$.

\subsection{Kick velocity of $Q N$}

Although it is clear that an SS produced by a QN is subject to kick velocity, our understanding of it is extremely limited. Based on the kick velocity of core collapse supernovae, one can assume that the distribution of kick velocity for QNs follows the Maxwell distribution:

$$
P\left(\nu_{\mathrm{k}}\right)=\sqrt{\frac{2}{\pi}} \frac{\nu_{\mathrm{k}}^{2}}{\sigma_{\mathrm{k}}^{3}} e^{-\nu_{\mathrm{k}}^{2} / 2 \sigma_{\mathrm{k}}^{2}}
$$

Furthermore, as stated in Ouyed et al. (2015), if a QN explosion is asymmetric, then it can provide a kick velocity of $100 \mathrm{~km} \mathrm{~s}^{-1}$. In order to facilitate discussion on the effect of kick velocities, this work takes $\sigma_{\mathrm{k}}$ as 50,100, 200 and $400 \mathrm{~km} \mathrm{~s}^{-1}$, respectively.

\subsection{Remnants of a $Q N$}

After a QN, the NS becomes a SS, which then gains kick velocity $\left(\sim 100 \mathrm{~km} \mathrm{~s}^{-1}\right)$ and loses mass $\left(\sim 10^{-3} \mathrm{M}_{\odot}\right)$ (Ouyed et al. 2015). This work assumes that the total mass lost in each QN is $10^{-3} \mathrm{M}_{\odot}$. As seen in Brandt \& Podsiadlowski (1995), certain kick velocities and lost masses can be used to calculate whether an LMXB has been disrupted or has survived post-QN. If the LMXBs is disrupted, then an isolated SS appears. If the spin period of the renascent SS is less than $30 \mathrm{~ms}$, then an IMSP is born. 
Furthermore, this work assumes that QNs do not alter the spin period of the entities involved. In other words, the spin period of a particular SS is presumed equal to that of its corresponding NS prior to the QN. As shown by the theoretical model of interaction between a rotating, magnetized NS and its surrounding matter (See: Pringle \& Rees 1972; Illarionov \& Sunyaev 1975; Ghosh \& Lamb 1978; Lovelace et al. 1995; Lü et al. 2012), the spin periods of an accreting NSs is dependent on both its mass-accreting rate and its magnetic field. After a sufficient mass transfer process has taken place, the spin period of an NS may reach an equilibrium spin period (Bhattacharya 1991). Based on Lipunov \& Postnov (1988), this can be given by

$$
P_{\mathrm{s}}^{\mathrm{eq}}=5.72 M_{\mathrm{NS}}^{-5 / 7} \dot{M}_{16}^{-3 / 7} \mu_{30}^{6 / 7} \mathrm{~s}
$$

when $\dot{\mathrm{M}}<\dot{\mathrm{M}}_{\text {Edd }}$ and

$$
P_{\mathrm{s}}^{\mathrm{eq}}=1.76 \times 10^{-1} \mu_{30}^{2 / 3} M_{\mathrm{NS}}^{-2 / 3} \mathrm{~s}
$$

when $\dot{\mathrm{M}} \geq \dot{\mathrm{M}}_{\mathrm{Edd}}$. Here, $\mu_{30}=\mu /\left(10^{30} \mathrm{G} \mathrm{cm}^{3}\right)$ is the NS's dipole magnetic momentum, $\dot{\mathrm{M}}_{16}=$ $\dot{\mathrm{M}}_{\mathrm{NS}} /\left(10^{16} \mathrm{~g} / \mathrm{s}\right)$ is its accretion rate and $\dot{\mathrm{M}}_{\mathrm{Edd}}$ is the Eddington accretion rate.

The magnetic field of an accreting NS has a direct impact on its spin period. Shibazaki et al. (1989) have suggested that accretion of mass may lead to the decay of an NSs magnetic field and proposed the following equation:

$$
B_{\mathrm{NS}}=\frac{B_{0}}{1+\triangle M / m_{\beta}}
$$

where $\triangle \mathrm{M}$ is the accreted mass, $\mathrm{m}_{\beta}$ is the mass constant for magnetic field decay, $\mathrm{B}_{\mathrm{NS}}$ is the field strength at time $\mathrm{t}$ and $\mathrm{B}_{0}$ is initial field strength of NS. Similar to Shibazaki et al. (1989), this work takes $\mathrm{m}_{\beta}=10^{-4} \mathrm{M}_{\odot}$ and $\mathrm{B}_{0}=10^{12} \mathrm{G}$.

\section{RESULT}

This paper uses population synthesis to simulate the $10^{7}$ binary system and, therethrough, investigate the total population of IMSPs produced by a particular QN. Our research team has used such methods widely in the past (See: Lü et al. 2006, 2008, 2009, 2012; Zhu et al. 2013). This work not only adopts Miller \& Scalo (1979)'s initial mass-function for the mass of primary components, but also a flat distribution of mass ratios (See: Kraicheva et al. 1989; Goldberg \& Mazeh 1994). In addition to this, this work also uses the distribution of separations of $\log \mathrm{a}=5 \mathrm{X}+1$, where $\mathrm{X}$ is a random variable uniformly distributed in the range $[0,1]$ and the separation a is in $\mathrm{R}_{\odot}$.

Our models have shown that IMSPs are a product of the disruption caused by QN on LMXBs. There are, however, some LMXBs that can avoid disruption and continue to survive post-QN. These include MSPs with a high eccentricity, called eMSPs.

\section{1. eMSPs}

Based on data taken from the ATNF pulsar catalogue database, there are a total of $25 \mathrm{MSPs}$ existing in eccentric binaries (ecc $>0.01)$ with low mass companion stars $\left(\mathrm{M}_{2} \leq 1 \mathrm{M}_{\odot}\right)$ (Manchester et al. 2005). Out of these 25, a total of 16 MSPs are in globular clusters. Since the high eccentricity of these MSPs is due to interactions with single stars(Rasio \& Heggie 1995; Bagchi \& Ray 2009), this paper focuses investigation on the remaining nine. Table 1 below gives a series of physical parameters for the nine MSP binaries in question. In general, MSPs produced through a recycling process are circular in orbit. The formation of eMSPs, however, is still highly debated. Freire \& Tauris (2014) have proposed that eMSPs may be formed as a result of the rotationally-delayed, accretion-induced collapse of a massive WD. Antoniadis (2014), on the other hand, states that the high eccentricity of MSPs may be a result of the dynamical interaction between the binary and the circum 
binary disk. Jiang et al. (2015) suggests that in LMXBs, the increase in mass of an accreting NS may result in an NS-SS phase transition, which then causes the NS to lose gravitational mass. This provides a satisfactory explanation for the origin of a number of eMSPs, including PSRs $\mathrm{J} 2234+06, \mathrm{~J} 1946+3417$ and $\mathrm{J} 1950+2414$, that have He WDs as companion stars. In recent literature, however, Stovall et al. (2019) has suggested that the formation of eMSPs may be a result of loss of mass during the proto-WD phase, where there is an unstable burning of shellhydrogen. The theoretical models used in this paper show that eMSPs originate from LMXBs that have survived post-QN, similar to the theory proposed by Jiang et al. (2015).In addition to this, our investigation has also brought kick velocity into consideration, which was a factor left unconsidered in previous studies.

If the binary system is undisrupted and continues to exist post-QN, then the additional kick velocity and loss of mass may lead to an orbital expansion. In our simulation model, the post-QN orbital parameters of an eMSPs binary system are determined by the standard formula given by Brandt \& Podsiadlowski (1995). As can be seen from Figure 1 below, our model can be applied to all eMSPs (when $\sigma_{\mathrm{k}}=50 \mathrm{~km} \mathrm{~s}^{-1}$ ), whereas other models are unable to explain the formation of MSP binaries with low eccentricity and low-mass companion stars. The reason for this is as follows: The lower the kick velocity, the more likely it is that the binary with low-mass companion stars will survive and the lower the eccentricity of the final surviving binary shall be.

\subsection{IMPSs}

If a QN disrupts an LMXB, then an IMSP will be born. In this paper, IMSPs are assumed to have masses of approximately $\sim 1.8 \mathrm{M}_{\odot}$. As far as current scholarship is concerned, however, it is impossible to measure the true mass of an IMSP. This paper also determines the spin pe-

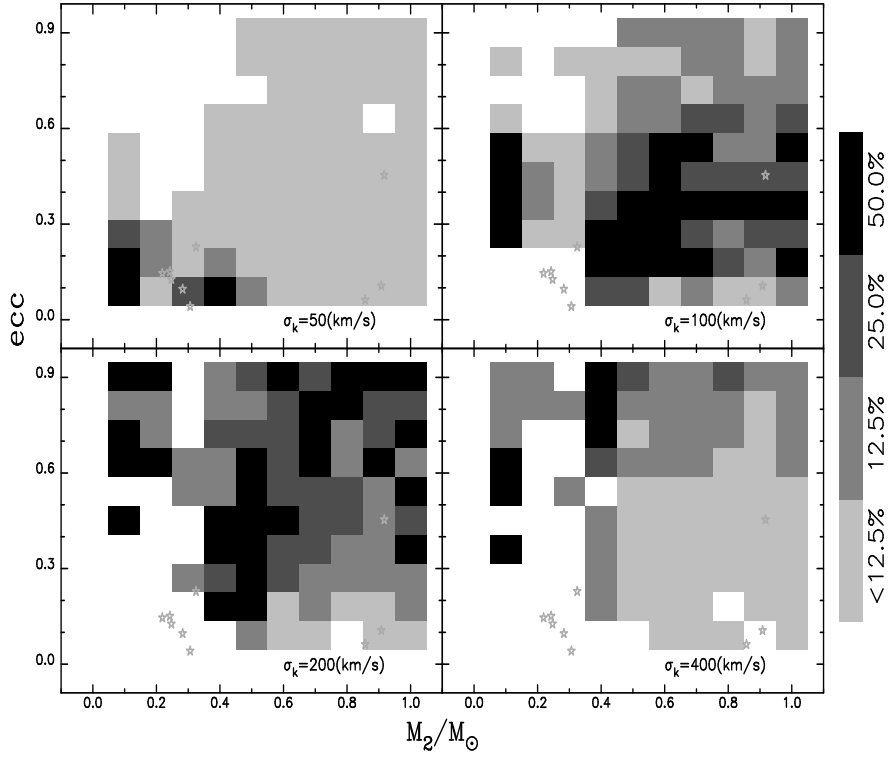

Figure 1. Grey-scale map of eccentricity and companion mass for MSP binaries that have survived post-QN. The various hues of grey correspond to the density of systems, within 1-1/2, 1/2$1 / 4,1 / 4-1 / 8$, and $1 / 8-0$ of the maximum $\frac{\partial^{2} N}{\partial e c c \partial M_{2}}$. Blank regions do not contain any stars. Stars represent the MSP binaries observed. Observational data was taken from the Pulsar Catalogue at ATNF(Manchester et al. 2005).

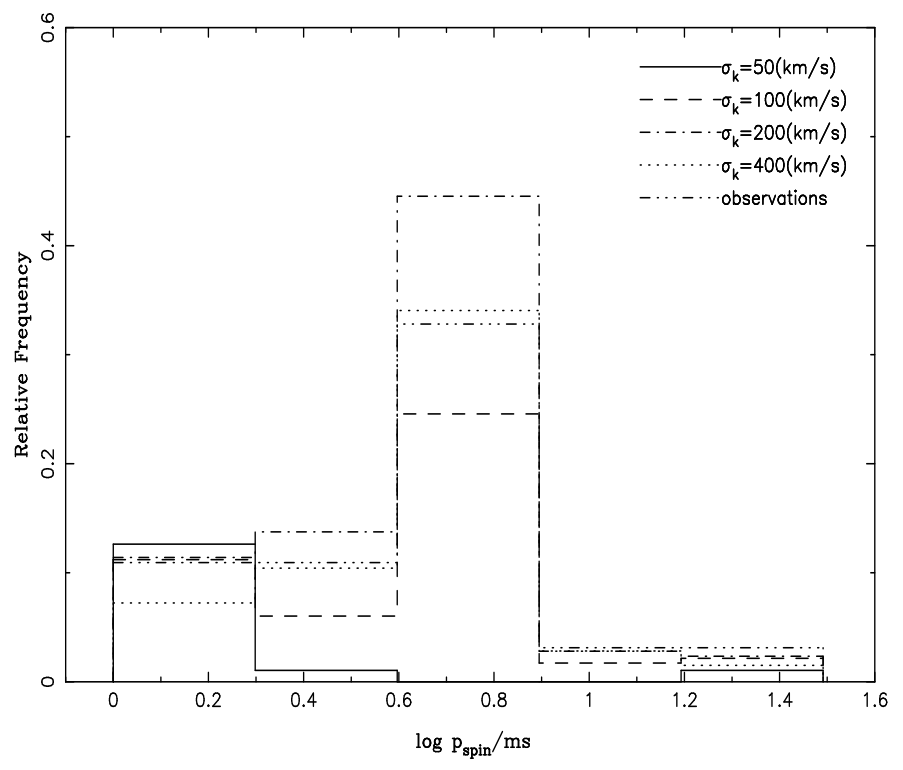

Figure 2. Distribution of QN-produced IMSPs spin periods with different kick velocities. The observed spin periods was taken from the Pulsar Catalogue at ATNF (Manchester et al. 2005). 
Table 1. Parameters for the nine observed eMSPS. The first column provides their names, the second column presents their orbital periods. The third column shows their spin periods and the fourth is the mass of the companion star. The eccentricity is given in the final column. Observational data was taken from the ATNF pulsar catalogue database(Manchester et al. 2005).

\begin{tabular}{lllll}
\hline \hline PSR Jname & $P_{\text {orb }}($ day $)$ & $P_{\mathrm{s}}(\mathrm{ms})$ & $M_{2}\left(M_{\odot}\right)$ & ecc \\
\hline $\mathrm{J} 0995-61$ & 24.578 & 1.99 & 0.22 & 0.11 \\
$\mathrm{~J} 1623-2631$ & 191.44 & 1.11 & 0.28 & 0.03 \\
$\mathrm{~J} 1727-2946$ & 40.30 & 2.71 & 0.83 & 0.05 \\
$\mathrm{~J} 1804-0735$ & 2.62 & 2.31 & 0.30 & 0.21 \\
$\mathrm{~J} 1903+0327$ & 95.17 & 2.15 & 0.89 & 0.44 \\
$\mathrm{~J} 1913+1102$ & 0.21 & 2.73 & 0.88 & 0.09 \\
$\mathrm{~J} 1946+3417$ & 27.01 & 3.17 & 0.21 & 0.13 \\
$\mathrm{~J} 1950+2414$ & 22.2 & 4.30 & 0.25 & 0.08 \\
$\mathrm{~J} 2234+0611$ & 32.00 & 3.58 & 0.19 & 0.13 \\
\hline \hline
\end{tabular}

riod of an IMSP using aforementioned equations (2) and (3). Figure 2 presents the spin periods of IMSPs produced by a QN, each with different kick velocities. According to the ATNF pulsar catalogue database, there are a total of 64 IMSPs in the galactic field (Manchester et al. 2005) and our results are consistent with this observation. Whats more, results have shown that the higher a QNs kick velocity is, the more easily IMSPs are produced. We estimate that the birthrate of QN-produced IMSPs as $\sim 9.5 \times 10^{-6} \mathrm{yr}^{-1}$ when $\sigma_{\mathrm{k}}=50 \mathrm{~km} \mathrm{~s}^{-1}$ and $\sim 1.7 \times 10^{-4} \mathrm{yr}^{-1}$, when $\sigma_{\mathrm{k}}=400 \mathrm{~km} \mathrm{~s}^{-1}$. Despite this, the majority of IMSPs are considered to be the result of the merging of an NS and a massive WD. The Galactic birthrate of IMSPs produced this way is between $\sim 10^{-4}$ and $10^{-6}$ $\mathrm{yr}^{-1}$ (Davies et al. 2002; Sun et al. 2018). Notably, our findings show that QN-produced IM-
SPs are statistically more significant than those produced by mergers.

The various theories for IMSP formation mentioned above are all related to binary MSPs, including the NS-WD merging theory (van den Heuvel \& Bonsema 1984), the NS disruption-recycling theory (Camilo et al. 1993), the triple-star system theory (Portegies Zwart et al 2011) and the QN formation theory presented in this work. However, as stated by Bailes et al. (1997) the luminosity of binary MSPs is different from that of others, such as PSRs J10240719, J1744-1134, J2124-3358, and J0711-6830. This suggests that a fraction of IMSPs may have been produced by another, entirely separate mechanism. In the majority of cases, the space velocity distribution of IMSPs is wider than that of binary MSPs (Lorimer et al. 2004). For this reason, IMSPs occupy a larger volume than binary MSPs and are more difficult to detect. Lorimer (2008) has suggested that there may even be more IMSPs in the Galaxy if ones takes this selection effect into account. In short, scholarship is still far away from fully understanding the origin of all IMSPs.

\section{CONCLUSIONS}

This paper has investigated QNs that occur in LMXBs when the accreting NSs mass reaches $1.8 \mathrm{M}_{\odot}$. If LMXBs are disrupted during this process, then IMSPs are produced. Using population synthesis, this paper estimated that the Galactic birthrate of QN-produced IMSPs is between $\sim 9.5 \times 10^{-6}$ and $\sim 1.7 \times 10^{-4} \mathrm{yr}^{-1}$. The uncertainties shown in our experimental model is due to the QNs kick velocity. Our findings show that QN-produced IMSPs are statistically more significant than those produced by mergers. The spin period of IMSPs is also consistent with the distribution given in previous observations. If the LMXBs are not disrupted and continue to survive after a $\mathrm{QN}$, then they may eventually become MSP binaries with a high eccentricity. 


\section{ACKNOWLEDGEMENTS}

We would like to thank the anonymous referee for their careful reading and constructive criticism. This work received generous sup- port from the National Natural Science Foundation of China, Project No. 11763007, 11863005, 11803026, and 11503008. We would also like to express our sincere gratitude to the Tianshan Youth Project of Xinjiang No. 2018Q014.

\section{REFERENCES}

Alpar, M. A., Cheng, A. F., Ruderman, M. A., \& Shaham, J. 1982, Nature, 300, 728

Antoniadis, J. 2014, ApJL, 797, L24

Backer, D. C. 1987, in IAU Symposium, Vol. 125, The Origin and Evolution of Neutron Stars, ed. D. J. Helfand \& J.-H. Huang, 13-21

Bagchi, M., \& Ray, A. 2009, ApJL, 693, L91

Bailes, M., Johnston, S., Bell, J. F., et al. 1997, ApJ, 481, 386

Belczynski, K., Lorimer, D. R., Ridley, J. P., \& Curran, S. J. 2010, Mon. Not. Roy. Astron. Soc., 407, 1245

Bhattacharya, D., \& Srinivasan, G. 1991, Journal of Astrophysics and Astronomy, 12, 17

Bhattacharya, D.van den Heuvel, E. P. J. 1991, PhR, 203, 1

Brandt, N., \& Podsiadlowski, P. 1995, MNRAS, 274,461

Camilo, F., Nice, D. J., \& Taylor, J. H. 1993, ApJL, 412, L37

Chen, H.-L., Chen, X., Tauris, T. M., \& Han, Z. 2013, ApJ, 775, 27

Cheng, K. S., \& Dai, Z. G. 1996, ApJ, 468, 819

Davies, M. B., Ritter, H., \& King, A. 2002, MNRAS, 335, 369

Ferrario, L., \& Wickramasinghe, D. 2007, MNRAS, 375, 1009

Freire, P. C. C., \& Tauris, T. M. 2014, MNRAS, 438, L86

Ghosh, P., \& Lamb, F. K. 1978, ApJL, 223, L83

Goldberg, D., \& Mazeh, T. 1994, A\&A, 282, 801

Grégoire, T., \& Knödlseder, J. 2013, A\&A, 554, A62

Hurley, J. R., Tout, C. A., \& Pols, O. R. 2002, MNRAS, 329, 897

Hutilukejiang, B., Zhu, C., Wang, Z., \& Lü, G. 2018, Journal of Astrophysics and Astronomy, 39,21

Illarionov, A. F., \& Sunyaev, R. A. 1975, A\&A, 39,185
Jiang, L., Li, X.-D., Dey, J., \& Dey, M. 2015, ApJ, 807, 41

Keränen, P., Ouyed, R., \& Jaikumar, P. 2005, ApJ, 618, 485

Kiel, P. D., \& Hurley, J. R. 2006, MNRAS, 369, 1152

Kluzniak, W., Ruderman, M., Shaham, J., \& Tavani, M. 1988, Nature, 334, 225

Kraicheva, Z. T., Popova, E. I., Tutukov, A. V., \& Yungel'Son, L. R. 1989, Astrophysics, 30, 323

Lipunov, V. M., \& Postnov, K. A. 1988, A\&A, 206, L15

Lorimer, D. R. 2008, Living Reviews in Relativity, 11, arXiv:0811.0762

Lorimer, D. R., Lyne, A. G., Bailes, M., et al. 1996, MNRAS, 283, 1383

Lorimer, D. R., McLaughlin, M. A., Arzoumanian, Z., et al. 2004, MNRAS, 347, L21

Lovelace, R. V. E., Romanova, M. M., \& Bisnovatyi-Kogan, G. S. 1995, MNRAS, 275, 244

Lü, G., Yungelson, L., \& Han, Z. 2006, MNRAS, 372,1389

Lü, G., Zhu, C., Han, Z., \& Wang, Z. 2008, ApJ, 683,990

Lü, G., Zhu, C., Wang, Z., Huo, W., \& Yang, Y. 2011, MNRAS, 413, L11

Lü, G., Zhu, C., Wang, Z., \& Iminniyaz, H. 2017, ApJ, 847, 62

Lü, G., Zhu, C., Wang, Z., \& Wang, N. 2009, MNRAS, 396, 1086

Lü, G.-L., Zhu, C.-H., Postnov, K. A., et al. 2012, MNRAS, 424, 2265

Manchester, R. N., Hobbs, G. B., Teoh, A., \& Hobbs, M. 2005, AJ, 129, 1993

Manchester, R. N., \& IPTA. 2013, Classical and Quantum Gravity, 30, 224010

Miller, G. E., \& Scalo, J. M. 1979, ApJS, 41, 513

Ouyed, R., Dey, J., \& Dey, M. 2002, A\&A, 390, L39 
Ouyed, R., Koning, N., \& Leahy, D. 2013,

Research in Astronomy and Astrophysics, 13, 1463

Ouyed, R., Leahy, D., \& Koning, N. 2015, Research in Astronomy and Astrophysics, 15, 483

Ouyed, R., Leahy, D., \& Koning, N. 2015, Res. Astron. Astrophys., 15, 483

Ouyed, R., Staff, J., \& Jaikumar, P. 2011, ApJ, 743, 116

Portegies Zwart, S., van den Heuvel, E. P. J., van Leeuwen, J., \& Nelemans, G. 2011, ApJ, 734, 55

Pringle, J. E., \& Rees, M. J. 1972, A\&A, 21, 1

Rasio, F. A., \& Heggie, D. C. 1995, ApJL, 445, L133

Rawley, L. A., Taylor, J. H., Davis, M. M., \& Allan, D. W. 1987, Science, 238, 761

Ruderman, M., Shaham, J., \& Tavani, M. 1989, ApJ, 336, 507

Rukeya, R., Lü, G., Wang, Z., \& Zhu, C. 2017, PASP, 129, 074201

Shibazaki, N., Murakami, T., Shaham, J., \&

Nomoto, K. 1989, Nature, 342, 656

Stappers, B. W., Bailes, M., Manchester, R. N., Sandhu, J. S., \& Toscano, M. 1998, ApJL, 499, L183
Stovall, K., Freire, P. C. C., Antoniadis, J., et al. 2019, ApJ, 870, 74

Sun, S., Li, L., Liu, H., et al. 2018, arXiv e-prints, arXiv:1812.02582

Sun, W., Zhu, C., Wang, Z., \& Lü, G. 2016, Ap\&SS, 361, 275

Tauris, T. M. 2016, Mem. Soc. Astron. Italiana, 87,517

van den Heuvel, E. P. J. 2008, in American Institute of Physics Conference Series, Vol. 983, 40 Years of Pulsars: Millisecond Pulsars, Magnetars and More, ed. C. Bassa, Z. Wang, A. Cumming, \& V. M. Kaspi, 554-557

van den Heuvel, E. P. J., \& Bonsema, P. T. J. 1984, A\&A, 139, L16

van den Heuvel, E. P. J., \& van Paradijs, J. 1988, Nature, 334, 227

Witten, E. 1984, PhRvD, 30, 272

Yisikandeer, A., Zhu, C., Wang, Z., \& Lü, G. 2016, Journal of Astrophysics and Astronomy, 37, 22

Zhu, C., Chang, P., van Kerkwijk, M. H., \& Wadsley, J. 2013, ApJ, 767, 164

Zhu, C., Lü, G., \& Wang, Z. 2015, MNRAS, 454, 1725

—. 2017, ApJ, 835, 249

Zhu, C., Lü, G., Wang, Z., \& Liu, J. 2013, Publ. Astron. Soc. Pac., 125, 25 